\title{
Fluorescent Metallosupramolecular Elastomers for Fast and Ultrasensitive Humidity Sensing
}

\author{
Miaomiao Li, ${ }^{\#}$ Quanqian Lyu, ${ }^{\#}$ Lvetao Sun, Bolun Peng, Lianbin Zhang*, and Jintao Zhu
}

Key Lab of Material Chemistry for Energy Conversion and Storage of Ministry of Education (HUST), School of Chemistry and Chemical Engineering, Huazhong University of Science and Technology (HUST), Wuhan 430074, China

\#These authors contributed equally to this work.

*E-mail: zhanglianbin@ @ust.edu.cn (Lianbin Zhang)

\section{Table of contents:}

Figure S1. Gel Permeation Chromatography curves of PTMG and PU . . . . . . . . . . . . . S-2

Figure S2. The chemical structures and 1H-NMR spectra of PU and PU-Eu . . . . . . . . . . . S-2

Figure S3. Rheological curves for PU and PU-Eu elastomer . . . . . . . . . . . . . . . . . . S-3

Figure S4. PL intensity and thickness of PU-Eu elastomer as a function of strains . . . . . . . . . S-3

Figure S5. Schematic illustration of the humidity chamber and lab-made setup for PL measurements • . S-4 Figure S6. Langmuir adsorption model for the water adsorption . . . . . . . . . . . . . . . . S-4

Figure S7. Transmittance spectra of elastomer before and after the irradiation of UV light . . . . . S-5

Figure S8. Preparation and characterization of CA membrane and CA/PU-Eu membrane . . . . . . . S-6

Figure S9. Comparison of recovery time of CA/PU-Eu membrane and PU-Eu elastomer . . . . . . . S-7 


\section{Supporting Figures:}

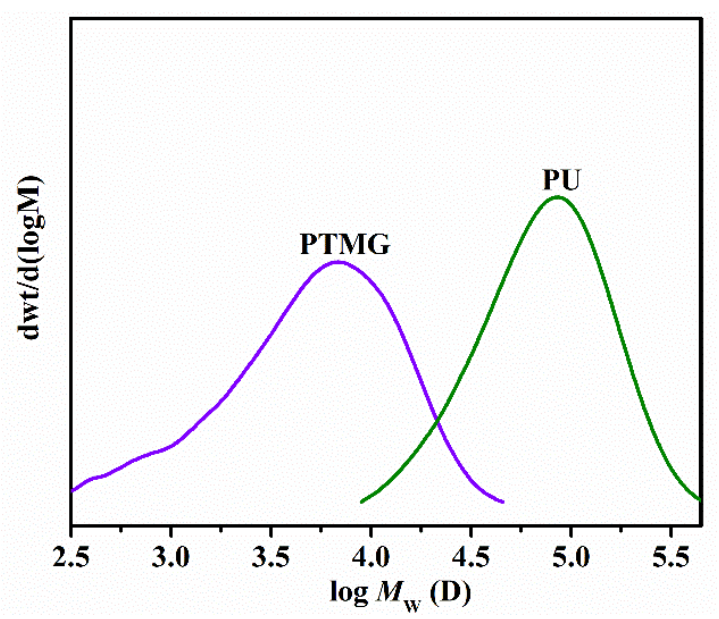

Figure S1. Gel Permeation Chromatography (GPC) curves of PTMG and PU.

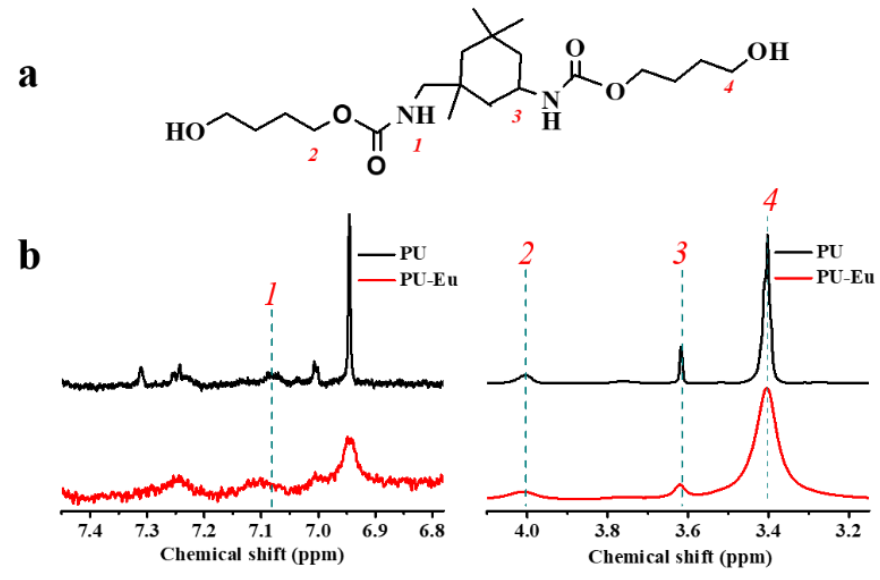

Figure S2. The chemical structures (a) and ${ }^{1} \mathrm{H}-\mathrm{NMR}$ spectra (b) of PU and PU-Eu in $d_{8}$-THF. 


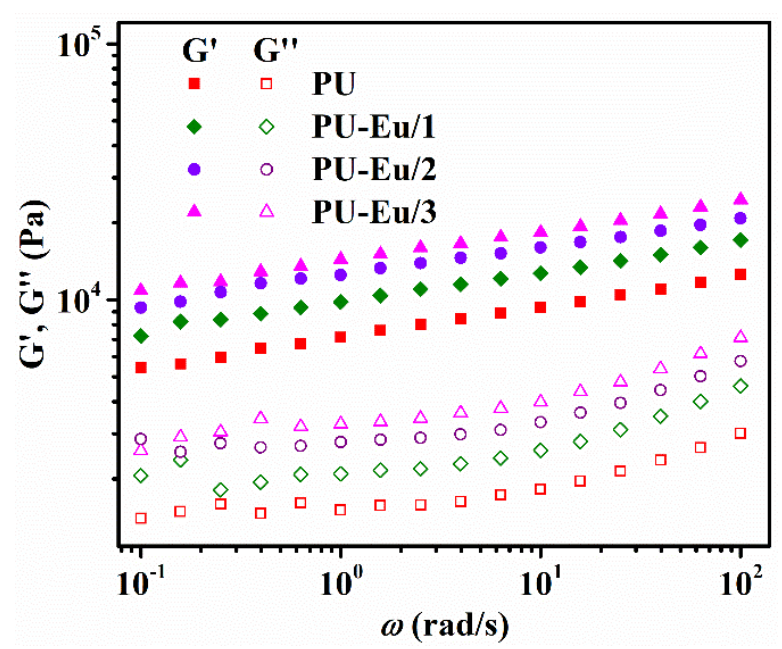

Figure S3. Rheological curves for PU and PU-Eu elastomer with different mole ratios of Eu-to-urethane (1:10, $1: 5$, and $1: 3)$.
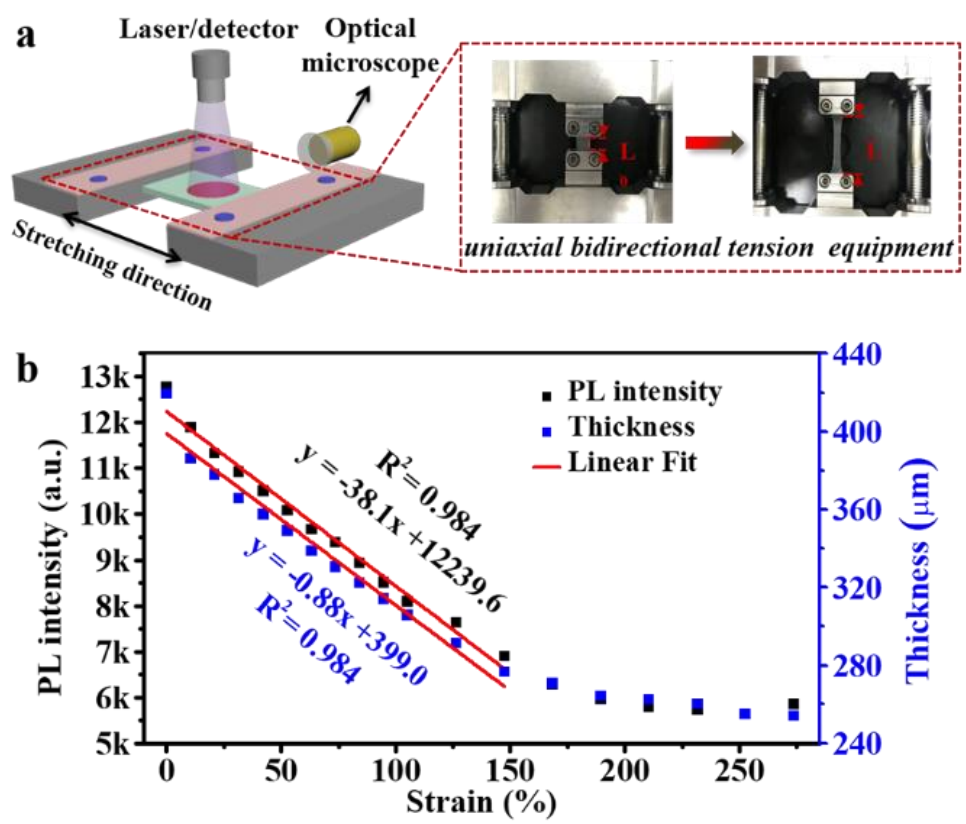

Figure S4. (a) Illustration of the lab-made mechanical and optical detection system and photographs of the original state and the elongated state of the PU-Eu elastomer with a strain of $275 \%$ under a uniaxial bidirectional tension device. (b) PL intensity and thickness of PU-Eu elastomer as a function of strains, and the linear fitted lines of the change in PL intensity and thickness. 


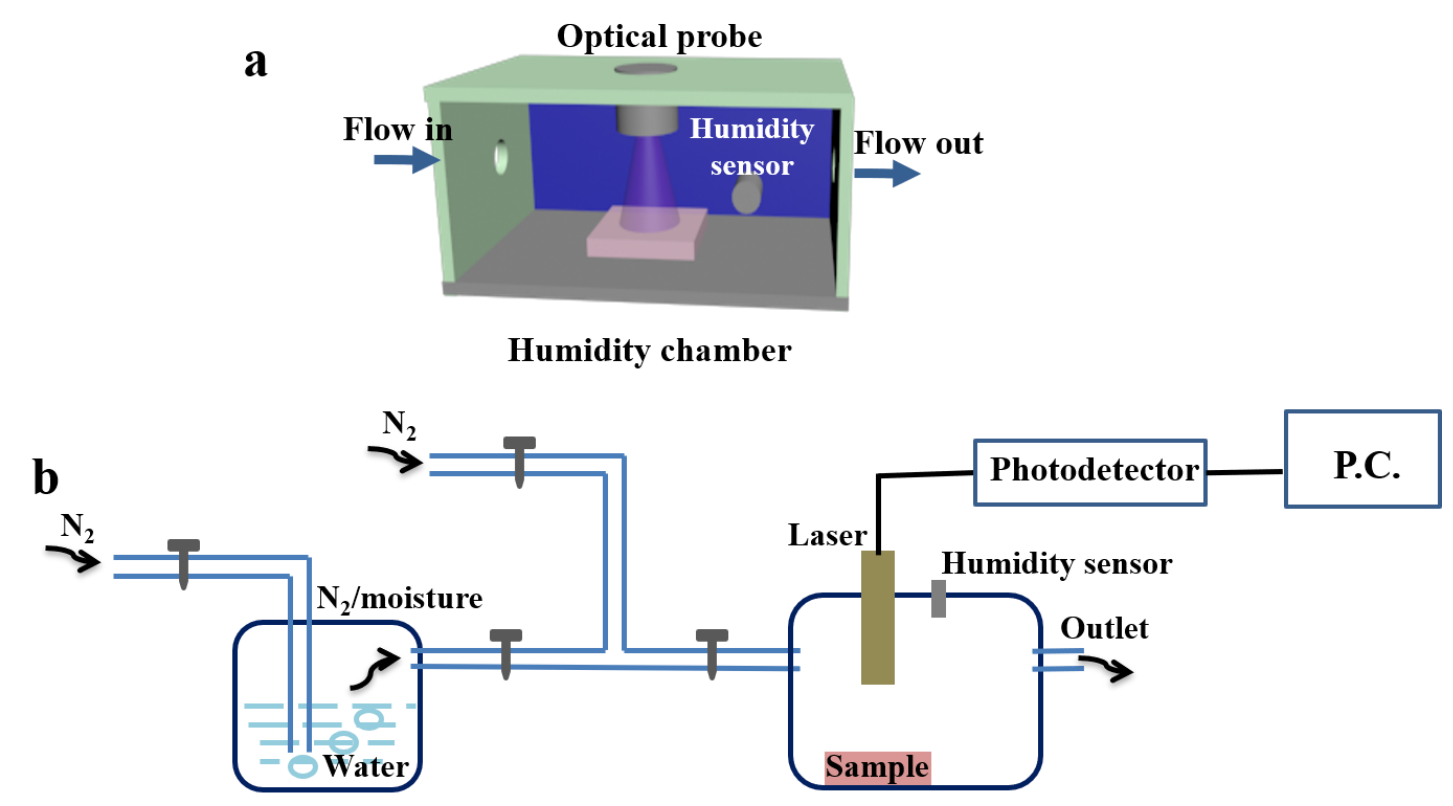

Figure S5. Schematic illustration of (a) the humidity chamber and (b) lab-made setup for PL measurements with controlled RH.

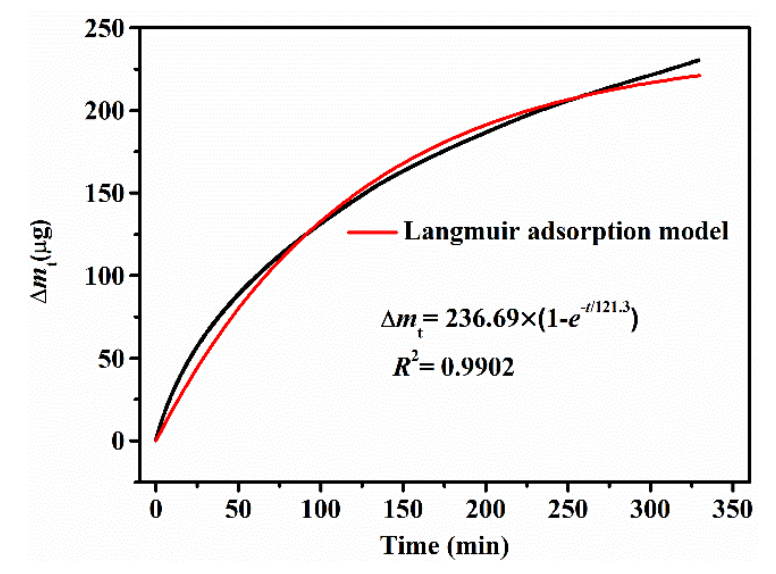

Figure S6. Langmuir adsorption model for the water adsorption of the PU-Eu elastomer at $20{ }^{\circ} \mathrm{C}$ and $93 \%$ RH.

The water adsorption $\left(\Delta m_{\mathrm{t}}\right)$ of PU-Eu elastomer was shown in Figure $\mathbf{S 3}$ and it can be described as following Equation S1: 1 , S2

$$
\Delta m_{\mathrm{t}}=\Delta m_{\infty}\left(A \quad e^{-t / \tau}\right)
$$


Where $\Delta m_{\infty}$ is the maximum of water adsorption, $\tau$ is relaxation time and relative to the adsorption and desorption rates and the mole concentration of water molecules. Then, the Langmuir adsorption kinetic model was used to fit the change in water adsorption. A Langmuir adsorption model can be established as $\Delta m_{\mathrm{t}}=59.2 \times\left(1-e^{-t / 121.3}\right)$, which shows a high correlation coefficient $(\sim 0.9902)$. The increased $23.7 \%$ of dry elastomer weight indicates that such a PTMG-based PU possesses good water adsorption characteristics, which is conducive for humidity sensing.

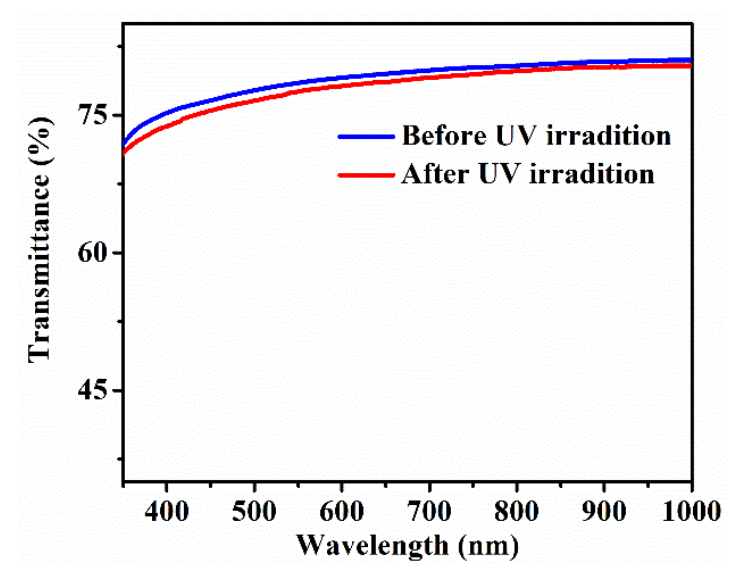

Figure S7. Transmittance spectra of PU-Eu elastomer with a thickness of $127 \mu \mathrm{m}$ before and after the irradiation of UV light for 12 hours. 

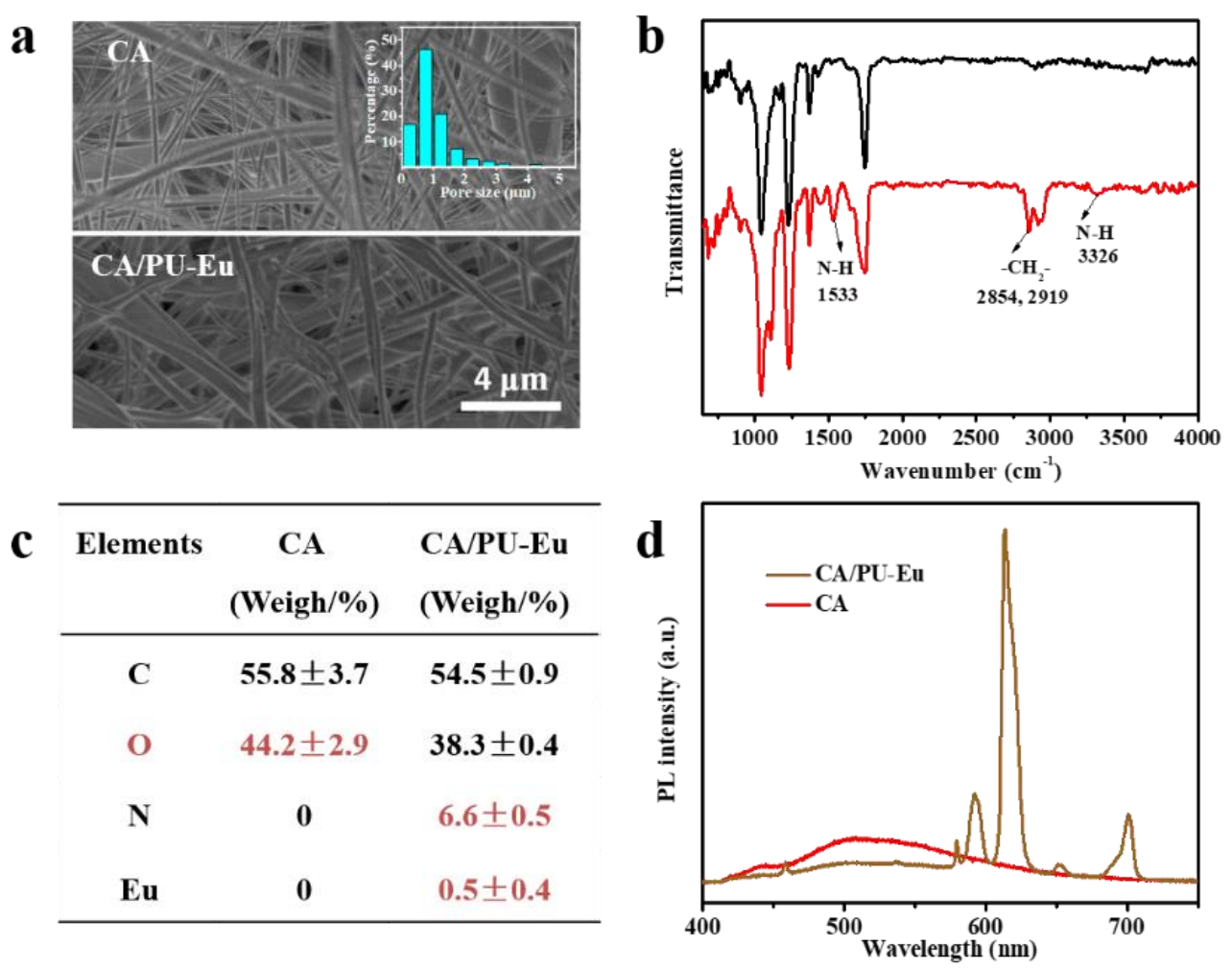

Figure S8. Preparation and characterization of CA membrane and CA/PU-Eu membrane. (a) SEM images of CA membrane (top) and CA/PU-Eu membrane (down). Inset (a): statistical two-dimensional pore distribution from the top SEM image of CA membrane. (b) FTIR spectra and (c) Energy dispersive spectroscopy (EDS) of CA membrane and CA/PU-Eu membrane. (d) PL spectra of CA membrane and CA/PU-Eu membrane.

We first examined the microstructures of CA membrane before and after coating PU-Eu elastomer by SEM investigation (Figure S4a). Through the statistical analysis from the SEM image of the electrospun CA membrane, we estimated the two-dimensional porosity of the CA membrane was $\sim 14 \%$, and these pores were mainly distributed in 0-1.5 $\mu \mathrm{m}$ (Figure S4a inset). After being coated with PU-Eu, the apparent porosity of the CA/PU-Eu membrane was reduced to $3 \%$. Both absorption peaks of $\mathrm{N}-\mathrm{H}$ and $-\mathrm{CH}_{2}$ - in FTIR spectrum and nitrogen $(\mathrm{N})$ and Eu elements in the EDS appeared, indicating the successful preparation of the CA/PU-Eu membrane (Figure S4b and c). ${ }^{\mathrm{S} 3, \mathrm{~S} 4}$ As revealed in the EDS elemental analysis of CA membrane in Figure S4c, oxygen with the high content $\sim 44.2 \%$ in CA membrane demonstrated excellent hydrophilic properties, improving their responsiveness to RH. Interestingly, although a small amount of PU-Eu coated on the CA 
membrane, the humidity sensing material still showed strong luminescence signals in the PL spectrum (Figure S4d) by adjusting the coordination ratio of Eu-to-urethane (1:3).

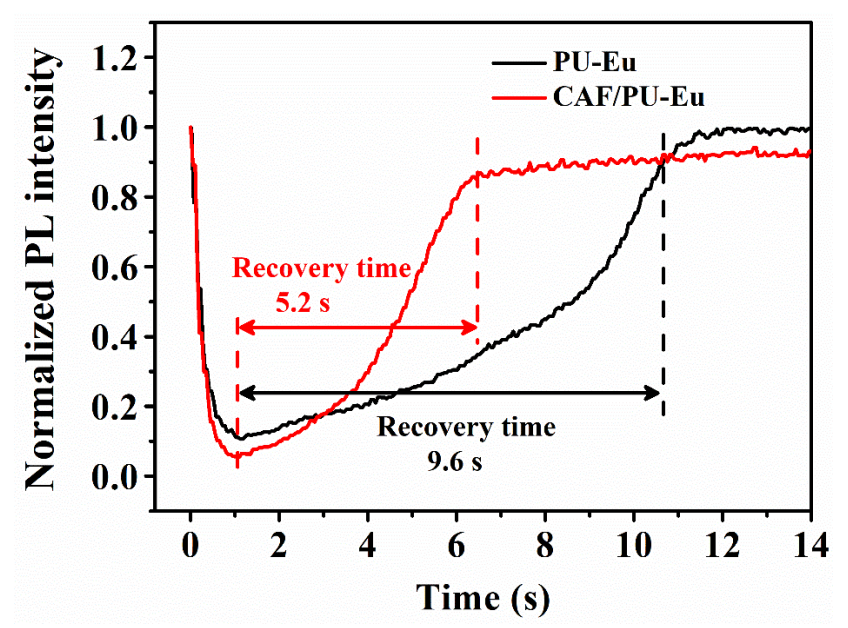

Figure S9. Comparison of the recovery time of the CA/PU-Eu membrane and PU-Eu elastomer for breathing sensing.

\section{Supplementary References}

(S1) Horzum, N.; Tascioglu, D.; Okur, S.; Demir, M. M. Humidity Sensing Properties of ZnO-Based Fibers by Electrospinning. Talanta 2011, 85, 1105-1111.

(S2) Zhang, D.; Wang, D.; Li, P.; Zhou, X.; Zong, X.; Dong, G. Facile Fabrication of High-Performance QCM Humidity Sensor Based on Layer-By-Layer Self-Assembled Polyaniline/Graphene Oxide Nanocomposite Film. Sensor. Actuat. B 2018, 255, 1869-1877.

(S3) Ma, L.; Wu, R.; Patil, A.; Zhu, S.; Meng, Z.; Meng, H.; Hou, C.; Zhang, Y.; Liu, Q.; Yu, R.; Wang, J.; Lin, N.; Liu, X. Y. Full-Textile Wireless Flexible Humidity Sensor for Human Physiological Monitoring. Adv. Funct. Mater. 2019, 29, 1904549-1904557.

(S4) Valente, A. J. M.; Polishchuk, A. Y.; Burrows, H. D.; Lobo, V. M. M. Permeation of Water as a Tool for Characterizing the Effect of Solvent, Film Thickness and Water Solubility in Cellulose Acetate Membranes. Eur. Polym. J. 2005, 41, 275-281. 